\title{
SPILLOVERS AND THE COST OF MULTIPROJECT R.\&D.
}

\author{
BY
}

PATRICK VAN CAYSEELE ${ }^{*}$

$D / 1985 / 2376 / 6$

*

I.C.M.-fellow, D.T.E.W., K.U. Leuven.

The author gratefully acknowledges the comments by $R$. De Bondt enabling substantial improvement upon earlier versions, as well as an enlightening discussion with H. Daems and P. De Grauwe. The usual caveat applies. 


\section{ABSTRACT}

The paper presents the costs of sequential and parallel research strategies in multiproject R.\&D. characterised by spillovers between the projects. Larger spillovers associated with a sequential strategy prove to be insufficient to guarantee a cost advantage for this option. This provides a rationale for a parallel research strategy with some duplication of effort, as observed in some real world R.\&D.-environments. 
INTRODUCTION

This is a paper on the optimal research strategy for a multiproject innovator. The R.\&D.-process has a time dimension and externalities exist between the different projects on the agenda. The available options are sequential or parallel completion of the projects. With sequential research the innovator tackles only one project at the time. After the completion of a project, knowledge useful in the completion of the next one becomes available. With parallel research the innovator tackles both projects simultaneously. knowledge overflows from one project on the other (spillovers) now realise immediately. As the full extent to which results from one project can be used for the completion of another only becomes clear after a while, some unnecessary duplication is inherent to parallel research. Hence the total amount of spillovers with parallel research is smaller than with sequential research.

We essentially show that this spillover advantage for a sequential strategy can be outweighted if knowledge accumulation is characterised by diminishing returns to time compression (Scherer (1967), Mansfield (1968)). This even holds for the extreme case in which no externalities between the projects exist under parallel research. Such a situation arises in international pharmaceutical drug development where parallel complete duplication of tests (knowledge) sometimes will be less expensive than sequential partial duplication. This odd result illustrates the main point of this paper: in reaching a conclusion regarding the optimal R.\&D.-strategy, a comparison of the total effort required will be often misguiding. The appropriate criterion is to be found in the costs of each strategy, which incorporate the dynamic features inherent to any R.\&D.-process.

\section{THE COSTS OF MULTIPROJECT RESEARCH AND DEVELOPMENT.}

In this section we derive the costs of completing two R.\&D.projects, both with a sequential and a parallel strategy. We then compare the costs and show under what conditions the spillover benefit of sequential research is insufficient to guarantee a cost advantage. Finally a specific case relevant to the pharmaceutical industry is studied.

\section{A. The Sequential Strategy}

Let $y(t)$ be the R.\&D.-expenditures at $t$ ime $t$, and let $x(t)$ stand for the cumulative knowledge reached on the project by $t$. The total knowledge required is $A$. The increase in knowledge $x(t)$ and the $R . \& D$-expenditures are related by ${ }^{1}$ ) 


$$
x^{\prime}(t)=y^{2}(t)(12) \quad x(0)=0(1 b) \quad x(T)=A(1 c)
$$

where $0<a<1$, reflecting the assumption of diminishing returns to spending money faster. As (la) indicates, the knowledge acquired in a period of time increases less than proportionally with increases in the research outlays for that period. In the production relation (la) - (lc), the completion date $T$ by which an amount of knowledge $A$ has been accumulated, can be viewed as the output. Then a cost minimising innovator will keep total discounted expenditures as small as possible while reaching $A$, or put formally he will solve (with $r$ the discount rate)

$$
\min _{y(t)} \int^{T} e^{-t} y(t) d t \quad(2)
$$

s.t. (la) - (lc)

It has been shown (Kamien and Schwartz (1972)) that the solution to this program is:

$$
C(T ; A)=\frac{A(A r r)^{1 / n}}{\left(e^{\pi T}-1\right)^{1 / n}}
$$

where $C(T ; A)$ is the least cost for completing an R.\&D.project requiring an amount of knowledge $A$ within a period of length $T$, and where $n=a /(1-a)$. A detailed derivation of a similar result is given below when we consider parallel research.

Now consider an innovator tackling two projects having completion dates $T_{1}$ and $T_{2}$ resp., and requiring resp. the amounts of knowledge $A_{1}$ and $A_{2}$. Without loss of generality, we assume $T_{1}<T_{2}$. The innovator tackles these projects simultaneously. When he has reached $A_{1}$ at $T_{1}$, this not only enables him to introduce the first invention, but this also provides him with a useful amount of knowledge $B$, for the completion of the second project. He therefore faces only the effort $A_{2}-B$, and has a $T_{2}-T_{1}$ period of time to come up with it. Formally, the cost function for sequential research will solve the program

$$
\begin{aligned}
& \min \left(\int e^{T_{1}}-\pi y_{1}(t) d t+\int^{T_{2}} e^{-\pi y_{2}}(t) d t\right) \\
& y_{1} y_{2}=0 \quad T_{1} \\
& \text { S.L. } x_{1}^{\prime}(t)=y_{1}^{a}(t) \quad(5 a) \quad x_{1}(0)=0 \quad(5 b) \quad x_{1}\left(\pi_{1}\right)=A_{1} \quad(5 c)
\end{aligned}
$$


5.t. $x_{2}^{\prime}(t)=y_{2}^{2}(t) \quad$ (6a) $\left.\quad x_{2}\left(\pi_{1}\right)=B \quad(6 b) \quad x_{2} \pi_{2}\right)=A_{2} \quad(6 c)$

The method developped by Kamien and Schwartz (1972) to solve problem (1a) $-(2)$, can be applied to $(4)-(6 c)$ in a straightforward way. We then obtain

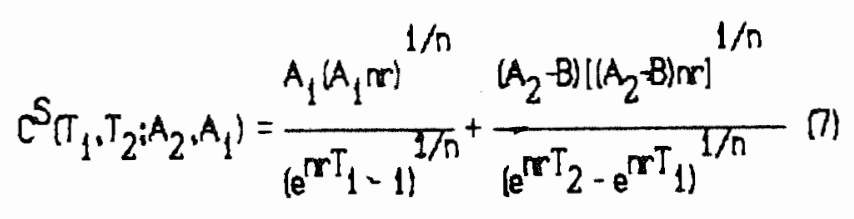

Equation ( 7 ) can also be found by simply substituting the appropriate efforts and completion times in the cost function displayed in expression ( 3 ). This follows from the possibility to decompose problem $(4)-(6 c)$ in two problems, each formally equivalent with problem (1)-(2).

\section{B. The Parallel strategy}

Consider again the innovator tackling two projects having completion dates $T_{1}$ and $T_{2}$ and requiring efforts $A_{1}$ and $A_{2}$. But now he follows a parallel research strategy, i.e. both projects are done simultaneously. From the donor project knowledge overflows (spillovers) contribute immediately to the completion of the receptor project. The donor project is identified as the one with the earliest completion date. The total amount of spillovers accumulated up to $T_{1}$ is assumed smaller than the one-shot lump sum spillover B, incurred at $\mathrm{T}_{1}$, when following a sequential strategy.

The problem for the multiproject innovator then can be formulated as follows:

$$
\begin{aligned}
& \min _{y_{1}, y_{2}}\left(\int_{1}^{T_{1}} e^{r t} y_{1}(t) d t+\int_{0}^{T_{2}} e^{r t} y_{2}(t) d t\right) \quad(8) \\
& \text { c.t. } x_{1}^{\prime}(t)=y_{1}^{a}(t) \quad(9 a) \quad x_{1}(0)=0(96) \quad x_{1}\left(T_{1}\right)(9 c) \\
& x_{2}^{\prime}(t)=y_{2}^{a}(t)+\varphi_{1}^{\prime}(t) \quad(10 a) \quad x_{2}(0)=0(106) \quad x_{2}\left(T_{2}\right)=A_{2} \quad(10 c)
\end{aligned}
$$

where q now denotes the spillover proportional to knowledge reached on the first project 
One now can show:

Proposition 1: The cost function for the parallel research strategy to complete 2 innovations with completion dates $T_{1}$ resp. $T_{2}$ and efforts $A_{1}$ and $A_{2}$ is

$$
C^{P}\left(T_{1}, T_{2} ; A_{1}, A_{2}\right)=\frac{A_{1}\left(A_{1} \pi\right)^{1 / n}}{\left(e^{\pi T} 1-1\right)^{1 / n}}+\frac{\left(A_{2}-q A_{1}\right)\left[\left(A_{2}-q A_{1}\right) r r\right]}{\left(e^{\left.\pi T T_{2}-1\right)^{1 / n}}(11)\right.}
$$

Proof:

The problem of finding an optimal path from 0 to $T_{2}$ is decomposed in finding an optimal path from 0 to $T_{1}$ and an optimal path from $T_{1}$ to $T_{2}$ given that the initial conditions of the latter form the endpoint conditions of the first, and need to be determined in an optimal way. The strategy is to start with the problem of reaching $T_{2}$ optimally assuming that one arrived already optimally in $T_{1}$ (part a). This yields a cost function in the remainder of time $\mathrm{T}_{2}-\mathrm{T}_{1}$, to acquire the remainder of knowledge $A_{2}-S$, where $S$ is the optimal amount of knowledge reached on the second project at $T_{1}$. Then we solve from 0 to $T_{1}$ and include this cost function as a salvage value (part b). This enables us to determine s explicitely, so as to obtain expression (11).

Part a) Solving the program

$$
\begin{aligned}
& \min ^{T_{2}} \int^{y_{2}} e^{-T t} y_{2}(t) d t \\
& \text { s.t. } x_{2}^{3}(t)=y_{2}^{a}(t)(13 a) \quad x_{2}\left(T_{1}\right)=5(13 b) \quad x_{2}\left(T_{2}\right)=A_{2} \quad(13 c)
\end{aligned}
$$

yields a cost function

$$
C\left(T_{2}-T_{1} ; A_{2}-S\right)=\frac{\left(A_{2}-S\right)^{\left(\left(A_{2}-S\right) r r\right]}}{\left(e^{\left.n T T_{2} \cdot \theta^{r T} T_{1}\right)^{1 / n}}\right.}
$$

for $(12)-(13 c)$ has the same structure as $(1 a)-(2)$. 
Part b) The next step is to solve

$\left.\left.\min _{y_{1}, y_{2}} \iint_{0}^{T_{1}} e^{-r t}\left(y_{1}(t)+y_{2}(t)\right) d t+C T_{2}-T_{1} ; A_{2}-S\right)\right]$

s.t. $(9 \mathrm{a}),(9 \mathrm{~b})$ and $(9 \mathrm{c})$

s.t. $(10 \mathrm{a}),(106)$ and $(136)$

We proceed by forming the Hamiltonian

$$
H \equiv-e^{-r t}\left[y_{1}(t)+y_{2}(t)\right]+\mu_{1}(t) y_{1}^{a}(t)+\mu_{2}(t)\left[y_{2}(t)+y_{1}^{a}(t)\right] \quad(17)
$$

and derive following necessary and also sufficient conditions (Seierstad and Sydsaeter (1977), Kamien and Schwartz (1981)).

$$
\begin{aligned}
& H_{y_{1}}=e^{-r t}+\mu_{1}(t) a y_{1}^{a-1}(t)+\mu_{2}(t) a y_{2} y^{-1}(t) \equiv 0 \\
& H_{y_{2}}=-e^{-r t}+\mu_{2} \text { (t)ay }_{2}^{a-1}(t) \equiv 0 \\
& -\mu_{1}^{\prime}(t) \equiv H_{x_{1}}=0 \\
& -\mu_{2}^{\prime}(t) \equiv H_{x_{2}}=0 \\
& H_{2}\left(T_{2}\right) \geq \frac{\delta C\left(T_{2}-T_{1}: A_{2}-S\right)}{\delta S}=\frac{n+1}{n} \frac{\left[\left(A_{2}-S\right) \mathbf{r r}\right]^{1 / n}}{\left(e^{\left.r T_{2}-e^{r \mathbf{r} T} 1\right)^{1 / n}}\right.} \\
& \left.x_{2} T_{1}\right)-q A_{1} \geq 0 \\
& \left(x_{2}\left(T_{1}\right)-q A_{1}\right)\left(\mu_{2}(t)+\stackrel{d C\left(T_{2}-T_{1} ; A_{2}-S\right)}{ }\right. \\
& \infty
\end{aligned}
$$

From (20) and (2l) it is clear that $\mu_{1}$ and $\mu_{2}$ are constants. Condition (22) has the following interesting economic interpretation: if the shadow cost of knowledge for the second project before the completion of the first exceeds the marginal cost of adding knowledge after completion ( $\mu_{2}$ ) $\delta \mathrm{C} / \delta \mathrm{S})$, the amount of knowledge reached on the second 
project at $T_{I}$ will be equal to the total amount of spillovers $\mathrm{qA}_{1}$. Intuitively it is clear that it will not be optimal to spent on research before $T_{1}$ if the resulting knowledge can be added more cheaply later on. Conversely, whenever there has been spending for research we have (22) holding with equality, a condition that will be useful in determining the optimal S.

We now derive the costs associated with the earliest project by starting from (18) and writing:

$$
y_{1}^{a}(t)=\left(\frac{e^{-r t}}{\left(\mu_{1}+\mu_{2} q\right) a}\right)^{a / a-1}
$$

From the boundary conditions we have

$$
\begin{aligned}
A_{1} \equiv x_{1}\left(T_{1}\right)-x_{1}(0)= & \int_{0}^{T_{1}} x_{1}^{\prime}(t) d t=\int_{0}^{T_{1}} y_{1}(t) t=\int_{0}^{T_{1}}\left(e^{t}\left(\mu_{1}+\mu_{2} q a\right)^{a / a-1} d\right. \\
& {\left[a\left(\mu_{1}+\mu_{2} q\right)\right]^{n}\left(e^{\left.m T_{1}-1\right)}\right.}
\end{aligned}
$$

$n$

from which an expression for $\mu_{1}+\mu_{2} q$ can be obtained, which after use in (25) yields

$$
y_{1}(t)=e^{r t / 1-a}\left(A_{1} n r\right)\left(e^{1 / a} T_{1}-1\right)^{-1 / a}
$$

This in turn can be used to obtain the cost function for the first project as

$$
C\left(T_{1} ; A_{1}\right)=A_{1}\left(A_{1} n\right)^{1 / a}\left(e^{m T} 1-1\right)^{-1 / n}
$$

We proceed by deriving the costs for working on the second project before $T_{1}$. Together with the cost for the work done after $T_{1}$, as obtained in part a), they constitute the entire cost for the last project. Taking up at (19) we write

$$
y_{2}^{a}(t)=\left(e^{r t}+2^{a}\right)^{a / a-1}
$$


Further, from the boundary conditions,

$$
\begin{aligned}
S \equiv x_{2}\left(T_{1}\right)-x_{2}(0) & =\int_{0}^{T_{1}} x_{2}^{\prime}(t) d t=\int_{0}^{T_{1}} \mid y_{2}(t)+q x_{1}^{\prime}(t) d t \\
& T_{1} a \\
& =\int_{2} y_{2}(t)+q A_{1} \\
0 &
\end{aligned}
$$

or

$$
S-q A_{1}=\int_{0}^{T_{1} a} y_{2}(t) d t=\int_{0}^{T}\left(e^{r t} \mu_{2} a\right)^{a / a-1} d t=\left(\mu_{2} a\right)^{n}\left(e^{r r T} 1-1\right) / r r
$$

from which we obtain

$$
\mu_{2}=\frac{\left[\left(S-q A_{1}\right)[r]^{1 / n}\left(e^{r r T} 1-1\right)^{-1 / n}\right.}{a}
$$

Expression (29) serves a double purpose. First it can be used to eliminate $\mu_{2}$ in (28), and second, in equation with the RHS of (22) it enables us to determinate $S$. Starting with the last task we have from equating (22) with (29)

$$
\begin{aligned}
& 1+n\left[\left(A_{2}-S\right) \pi\right]^{1 / n} \quad\left[\left(S-q A_{1}\right) n\right]^{1 / n} \\
& \text { n } \frac{}{\left(e^{m T_{2}}-e^{m T} 1\right)^{1 / n}}=\frac{}{a\left(e^{m T} T_{1}-1\right)^{1 / n}}
\end{aligned}
$$

which can be rearranged into

$$
S \equiv \frac{\xi}{1+\xi} A_{2}+\frac{1}{1+\xi} q A_{1}
$$

where $\frac{\left(e^{\mathrm{nr} T} 1-1\right)}{\left(e^{\mathrm{rr} T} 2-e^{\mathrm{rr} T_{1}}\right)} \equiv \xi$

(31)

Expression (30) shows that the amount of knowledge reached by the second project at the finishing date of the first is 
a weighted average between the total knowledge needed, and the knowledge spillover from the first project. The weights are given by the discount factors for the first time period $\left(0-T_{1}\right)$ and for the remaining time period $\left(T_{2}-T_{1}\right)$, relative to the entire time period $\left(0-T_{2}\right)$. These discount factors incorporate both time preference (cfr. the discount rate $r$ ) and compression diseconomies (cfr. n). Note that for finite introduction times of the second project and noninstantaneous completion of the earliest project, we have $S$ $>\mathrm{qA}_{1}$.

Since by now we have expressions both for $\mu_{2}$ and $S$, we can start from raising both sides of (28) to the power $1 / a$ and substituting for $\mu_{2}$ in order to obtain the optimal R.\&D. expenditure path for research outlays on the second project before $T_{1}$ as

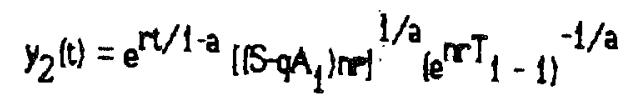

Integration of these expenditures appropriately discounted yields the costs for working on the second project before the completion of the first as

$$
C\left(T_{1}: S\right)=\left(S-q A_{1}\right)\left(\left(S-q A_{1}\right)(\mathbf{r}]^{1 / n}\left(e^{r r T} 1-1\right)\right.
$$

In (14) and (33), s can be replaced by the expression in (30). Adding together this gives the entire costs for completing the second project. Together with (27) this gives the cost function for parallel research as

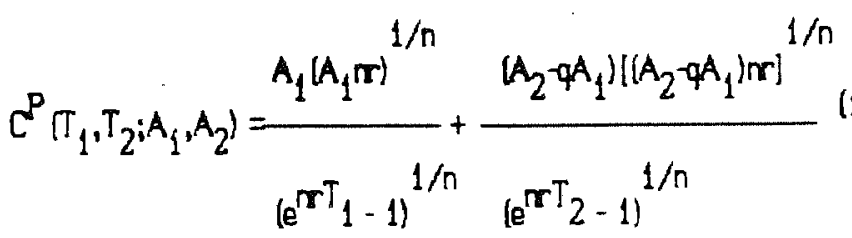

Q.E.D.

\section{The Strategies Compared}

Whether the multiproject innovator will engage in sequential or parallel research now will depend on whether $C^{s}<C P$ (or vice versa). An explicit comparison of equations (7) and (11) shows that this depends on whether 


$$
\frac{\left(A_{2}-B\right)^{n+1}}{\left(\theta^{n T} 2-\theta^{n T T_{1}}\right)}>\frac{\left(A_{2}-q A_{1}\right)^{n+1}}{\left(\theta^{n r T} 2-1\right)}
$$

A comparison of the nominators of both sides of (34) indicates less effort with a sequential strategy for $B>$ $\mathrm{qA}_{1}$, as waiting prevents unnecessary duplication. The one shot lump sum spillover however takes place after introduction of the first innovation resulting in less time $\left(T_{2}-T_{1} v i z . T_{2}\right)$ to complete the second project, as reflected by the denominators. This directly illustrates the main point of the paper: while a simple comparison in terms of total efforts (knowledge) accords an advantage to the sequential strategy $\left.\left(\mathrm{A}_{2}-\mathrm{B}\right)<\mathrm{A}_{2}-\mathrm{q}_{1}\right)$, this is not necessary translated into a cost advantage. Only if inequality (35) holds, sequential research will be the best strategy.

$$
\frac{\left(B-q A_{1}\right)}{\left(A_{2}-q A_{1}\right)}>\left(\frac{\left.\mid e^{r T T} 2-e^{r T T} 1\right)}{\left(e^{\operatorname{rrT}} 2-1\right)} t^{1 / n+1}-1\right.
$$

Basically this expression says that the relative advantage of sequential over parallel research i.e. $\left(B-q A_{1}\right) /\left(A_{2}-q A_{1}\right)$ has to be big enough to outweigh the time compression diseconomies associated with the shorter time period for completing the remaining effort. Indeed (35) is likely to be violated if the spillover advantage is small (too little) and/or the first introduction releasing the one shot spillover shortly preceeds the second (too late).

We can also make this point graphically. Then we represent the different options in the cost-output ( $t$ ime)-space. Since the derived costs are decreasing and convex functions in the length of the completion period, and increase with knowledge required, the following picture is justified: 
Fig. 1: The Costs of Sequential and Parallel strategies.

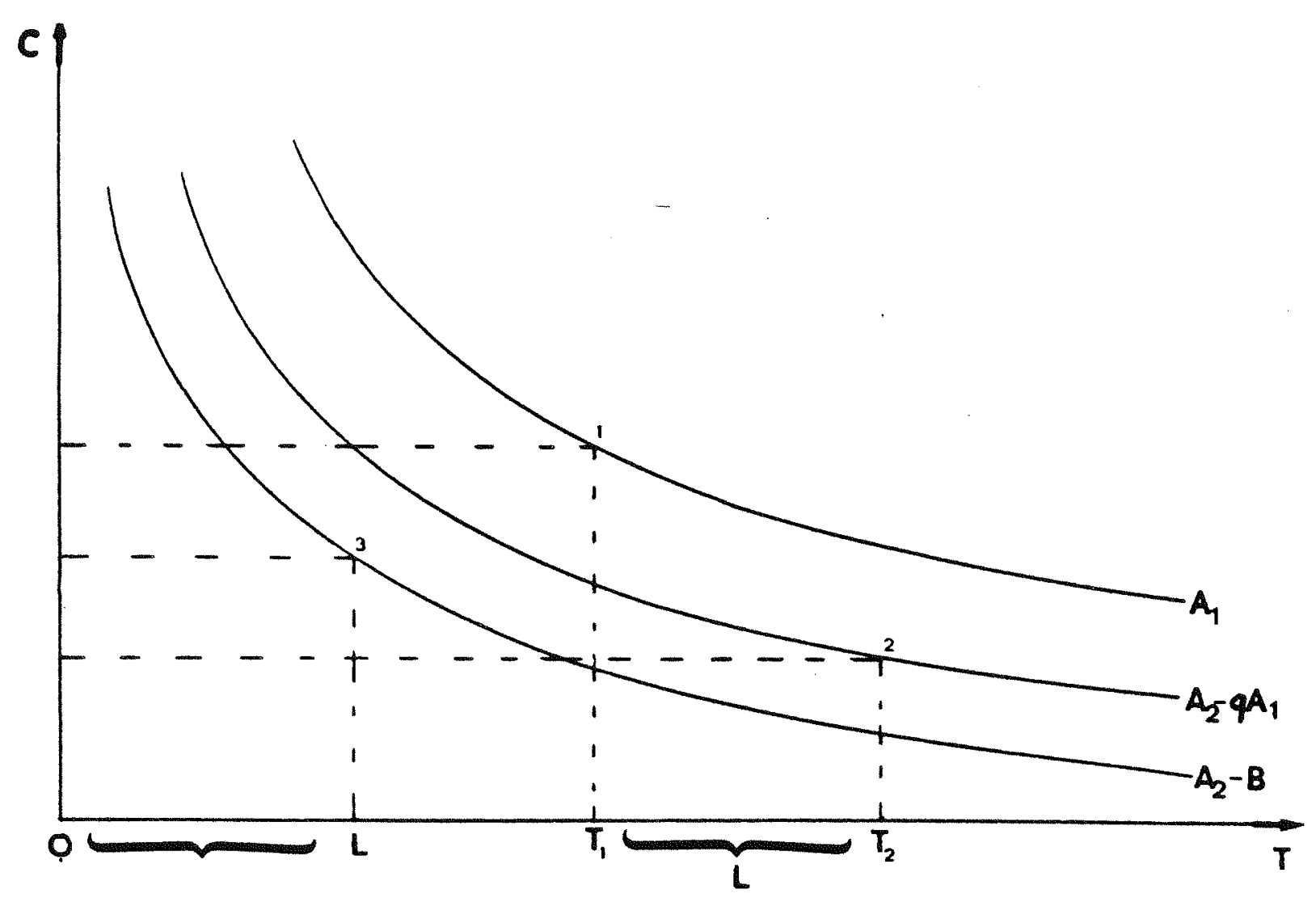

Point 1 gives the costs of completing the first project by $T_{1}$. This one is the same for both strategies. Point 2 gives the costs for completing the second project following the parallel strategy while point 3 shows these costs following the sequential strategy. We find these costs on a curve lying below that of parallel research (recall we assume $B$ ) $\mathrm{qA}_{1}$ ), but only $L=\mathrm{T}_{2}-\mathrm{T}_{1}$ periods away from the origin, not $\mathrm{T}_{2}$ ! Given that the costs of generating a certain amount of knowledge increase more than proportionally as the available time span decreases, one can easily imagine the costs of sequential research being higher. This is the case for the situation displayed in figure 1 . Of course, different values of the parameters underlying the R.\&D.-proces and/or different introduction dates lead to different outcomes. Therefore a last issue to adress is: which are the characteristics of an R.\&D.-environment in which parallel strategies thrive? As the costs for completing the first project are unaffected by the chosen option we can focus on the costs for the second project in answering this question. 
For the purpose we define

$$
W=\frac{\left(A_{2}-B\right)\left[\left(A_{2}-B\right)\left(e^{\pi T} 2-1\right)\right]^{1 / n}}{\left(A_{2}-q A_{1}\right)\left[\left(A_{2}-q A_{1}\right)\left(e^{\pi T} 2-\theta^{\pi T T_{1}}\right)\right.}
$$

An increase in $W$ will reflect a trend toward the choice of a parallel strategy for $W$ is nothing else than the ratio of the costs of completing the second project with a sequential strategy over the same cost with a parallel strategy. A comparative static analyses reveals:

Proposition 2 : Parallel research as compared with sequential becomes cheaper as there are decreases in the one shot sequential spillover $B$, in the discount rate $r$, in the completion date of the second project $T_{2}$, and as there are increases in the instanteneous parallel spillovers $q$, in the effort needed to complete the first project $A_{1}$, in the effort needed to complete the second project $A_{2}$ and in the introduction date of the first project, $T_{1}$.

Table 1: Comparative Statics: The relative cost of parallel vs. sequential research.

\begin{tabular}{llllllll} 
Par. & B q & \& & \& & $T_{1}$ & $T_{2}$ \\
Sign BW/Arar. & + & + & + & - & + & + \\
\hline Regult \# & 12 & 3 & 4 & 5 & 6 & 7
\end{tabular}

\section{Proof:}

By differentiation of $W$.

All these results are intuitively clear. Results 1,2 and 3 follow from the fact that increasing the spillovers for one option reduces its' costs. Result 4 follows from the fact that an increase in $A_{2}$ can be "spread out" over a larger period with parallel research than it can be with a sequential strategy, so that the resulting compression diseconomies will be bigger for this strategy. An increase in the discount rate raises the cost of money spent early. As one immediately starts spending on the second project under a parallel strategy, hence result 5. Finally results 6 and 7 follow from resp. squeezing and loosening the period 
$T_{2}-T_{1}$ in which $A_{2}-B$ has to be done. As $T_{1}$ increases this period is shortened resulting in more important time compression diseconomies. As $T_{2}$ increases the opposite holds for more time becomes available.

\section{A Specific Example}

Consider the institutional background of international pharmaceutical R.\&D. If a drug innovator plans clinical testing in a country, he has to submit first the results of other research, e.g. on pharmacokinetics and toxicology. Usually the results of research conducted abroad will be accepted for this purpose. This is not the case for clinical trials which often need to be done by local experts (IFPMA (1975)). But several regulatory requirements delay the transfer of foreign test results and by the same token belate the local clinical trials. Without going into the institutional details of all the different laws regulating the introduction of a drug, we nevertheless offer following causes:

- often a foreign licencing authority requires the results of domestic clinical trials before it allows to carry out the necessary local trials (IFPMA (1975));

- if the transfer is by means of expert testimonials or bibliografies, the domestic registration certificate sometimes has to be attached (Royal Decree on the Registration of Drugs in Belgium (1975)) 4 );

- apart from regulatory requirements in the foreign country, as the two just mentioned, also domestic regulators can delay foreign testing, such as the Food and Drug Administration (U.S.A.) requiring that the new chemical entity has obtained a domestic IND-approval before it can be exported, even for testing purposes (U.S. Department of Commerce (1983)).

In the sequel we assume that clinical drug testing abroad starting from domestic pharmacokinetic and toxicological knowledge is impossible as long as domestic registration hasn't been obtained. Thus market licencing authorities require that at least part of the tests are duplicated locally and delay the transfer of knowledge necessary to start local testing.

To avoid such lags the innovator can create the necessary results to start clinical trials by local research on pharmacokinetics and toxicology. Then each result reached on the project before $T_{1}$ will be an exact duplication of a spillover result becoming available on $T_{1}$. Thus not only the tests which have to be done locally but also those that could have been transferred will be duplicated. Spillovers with parallel research now will be completely unexisting, not simply smaller in comparison to a sequential strategy. The question now is whether parallel complete duplication can be less expensive than sequential partial duplication. The situation described can be analysed in the above 
framework by putting $q=0$. The equivalent of condition (35) necessary to guarantee a sequential strategy simply becomes:

$$
\underset{A_{2}}{B}\left[\frac{\left[e^{\pi r T_{2}-a^{\pi r T}} 1\right)}{\left(e^{\pi T} T_{2}-1\right)}\right\}^{1 / n+1}
$$

As the inequality sign in (36) is reversed, and apparently this doesn't contradict any of the assumptions, parallel complete duplication becomes the cheapest strategy.

In circumstances when (36) holds, the technology transfer takes place sequentially. This appears to be typical for German pharmaceutical enterprises who, as Heiduk (1981) shows, usually patent a molecule, develop it into a drug, introduce it domestically, registrate it abroad perhaps after some additional testing, and start exporting it to foreign affiliates distributing it. If the reverse conditions hold, we would expect enterprises to duplicate efforts in the countries in which the drug will be introduced. Such a pattern shows for France and Italy, countries that take extreme attitudes in disregarding the results from foreign tests. In these countries the affiliates of German pharmaceutical multinationals are fully integrated with research facilities and inland expertise, and they develop and manufacture the drug fully on their own ${ }^{5}$.

Also, as R.\&D.-decentralisation can be associated with a larger amount of parallel complete duplication than R.\&D.centralisation which typically will involve more of sequential partial duplication, we expect more overseas or foreign R.\&D.-expenditures in the pharmaceutical industry. Recent empirical analyses has revealed such trends (Mansfield et al. (1928)). One of the observed relations was
$\mathrm{a}^{\mathrm{i} \mathrm{t}}=-1.13+$
$0.73 \mathrm{~T}^{\mathrm{t}}$
$+0.15 Q^{i t}$
$+0.004 \mathrm{sit}^{\mathrm{it}}$
(3.09)
$+16.10 D_{i}$ $(0.44) \quad(0.34)$

$$
(3.09)
$$

with $a^{i t}$ the percentage of overseas R.\&D. in the enterprise in a given year.

$T^{t}$ a time dummy variable for some years.

$Q^{i} t$ the percentage of foreign sales on the enterprise in a given year.

$S^{i t}$ the sales of the enterprise in a given year.

$D^{1}$ a dummy that equals $l$ if the enterprise is a pharmaceutical company, and 0 otherwise.

As can be seen, the share of R.\&D.-expenditures carried out overseas is explained by the importance of foreign sales, and strongly and significantly by a dummy variable for the pharmaceutical nature of the company. It then is important to know that decentralisation should not be seen as a response to legal limitations on the transfer of knowledge 
only, but also as a result of the time compression diseconomies induced by regulatory lags.

\section{CONCLUSIONS AND RELATED ISSUES}

Earlier work on the optimal allocation strategy for multiple R.\&D. projects (Gittens (1972), Laska et al. (1972)) focussed on the stochastic characteristics of the process. If the success probabilities increase in the amount of resources devoted to the project, a sequential strategy has been identified as optimal. When these probabilities decrease, it is better to work simultaneously. In this paper, the optimal strategy for multi-project R.\&D. was studied in view of the spillover characteristics of the proces. It was shown that in certain environments a strategy of simultaneous research with partial or even complete duplication of one's own efforts can be optimal.

Recent work on spillovers focusses on the inappropriability disincentives to innovative activities induced by the involuntary dissemination of R.\&D.-results. These knowledge overflows between rival enterprises are externalities at the level of the market and are called upon to justify public intervetions to stimulate industries' dynamic performance (Hartwick (1984), spence (1984)). Here we studied spillovers that exclusively exist between research projects completed by a single innovator. This type of spillovers doesn't exclude the other, but has entirely different public policy implications.

If for example the creation of a new technology generates a lot of results that can be used for a future project, one cannot assume that enterprises don't take this into account. And this in turn implies that one cannot infer from the anticipated losses of a single project that an innovator intends to engage in predatory actions. This line of reasoning emerges in a recent analyses of the U.S. vs. I.B.M.-case (Pittman (1984)) $)^{6}$. As similar future cases possibly also will call upon these intrafirm spillovers, and as they are likely difficult to quantify, we think it is important to know that these spillovers do not translate theirselves automatically into lower costs.

\section{Notes}

1) Following notational conventions are valid throughout the paper:

- an accent after, rather than a dot above, a variable denotes its' differentiation w.r.t. time, e.g.

$$
-\quad x^{2}(t)=\delta(t) / d t
$$


- the same holds for differentiation with respect to any other variable, which now will follow the function explicitely, e.g.

$$
H / \delta y_{1}=H_{y_{1}}
$$

- in order to indicate partial differentiation the $\delta$ of variational calculus is used.

2) One can also view this spillover as proportional to the effort required for the first project and write.

$$
B=\nu A_{1}
$$

This is closer to the formulation parallel strategy, where we have of spillovers with a $q A_{I}$. One then assumes.

\section{$v>q$}

3) As we assume $T_{1}<T_{2}$, this implies that spillovers go from project 1 to project 2 . If we assume $T_{1}>T_{2}$ and maintain the assumption that spillovers go from the project with the earliest completion date to the latest, we obtain cost functions which are symmetric with (11). One only has to interchange the subscipts 1 and 2 . Mathematically nothing changes. If spillovers are associated with one project, e.g. they go from project 2 on project 1 , regardless the sequence of completion dates, and not vice versa, a solution with a mathematical structure different from (11) will be obtained for the case $T_{1}<T_{2}$. The fact that the amount of spillovers varies proportionally with the state of knowledge reached on the second project at $\mathrm{T}_{1}$ increases the complexity significantly. In this paper we will not explore this strand of research.

4) Belgian authorities do not refuse foreign clinical data but rather make objections toward analytical and stability data. A local control of analytical methods must demonstrate that they can be used (IFPMA (1975)).

5) This might explain why affiliates of one enterprise sometimes end up with different drugs. As Heiduk (1981) notes:

"These characteristics prove that affiliated companies of German pharmaceutical concerns in France and Italy are rather a reflection of the structure of the parent companies - although in part with a different product sortiment - whereas, in other countries, predominantly partial functions are carried out".

6) More precisely, Pittman (1984) says:

"In any industry in which technological change in rapid, 
the benefits of the development of a new product are likely to include improvements in other products sold by the firm and information which leads to the developmentof future products. These 'externalities' - external to the particular product line, internal to the firm - are likely to be difficult to quantify. Nevertheless, a rational firm must take them into account in its investment planning, and an analyst testing for predatation must include them in the benefits of a program". The author argues that in the I.B.M.-case spillovers probably did not compensate the losses of the project,but dismisses predatory behavior for other reasons.

Bibliography

Gittens J.; 1972, Some Problems of Stochastic Ressource Allocation, Journal of Applied Probability 9, pp. 360-369.

Hartwick J.; 1984, Optimal R.\&D. levels when Firm j benefits from Firm i's inventive activity, Economic Letters 16, pp. $165-170$.

Heiduk G.; 1981, Multinationalisation in the Pharmaceutical Industry as a Response to National Health Policies, in European Association for Research in Industrial Economics, Proceedings of the $8 \mathrm{th}$ Conference, Basel.

I.F.P.M.A.; 1975, National Regulations for Pharmaceutical Drug Introduction, $295 \mathrm{p}$.

Kamien $M$. and $N$. Schwartz; 1972, Timing of Innovations under Rivalry, Econometrica 40 (1), pp. 50-51.

Kamien M. and N. Schwartz; 1981, Dynamic Optimisation: the Calculus of Variations and Optimal Control in Economics and Management, North-Holland, Amsterdam, $331 \mathrm{p}$.

Kamien $M$, and N. Schwartz; 1982, Market structure and Innovation, Cambridge University Press, Cambridge.

Laska E., M. Meisner and C. Siegel; 1972, Contributions to the Theory of Optimal Ressource Allocation, Journal of Applied Probability 9, pp.

Mansfield E.; 1968, The Economics of Technological Change, W.W. Norton, New York. 
Mansfield et al.; 1982, Technology Transfer, Productivity, and Economic Policy, W.W. Norton, New York.

Pittman R.; 1984, Predatory Investment: U.S. vS. I.B.M., International Journal of Industrial organisation 2, pp. 341365 .

Spence A.M.; 1984, Cost Reduction, Competition and Industry Performance, Econometrica 52 (1), pp. 101-121.

Royal Decree on the Registration of Drugs; 1969, ch. I, art. 8 \& 10, Belgisch Staatsblad, 15-10-'75.

Scherer F.M.; 1967, Research and Development Resource Allocation under Rivalry, Quarterly Journal of Economics 81 , pp. 359-394.

Seierstad A. and K. Sydsaeter; 1977, Sufficient Conditions in Optimal Control Theory, International Economic Review 18 , pp. $367-391$.

U.S. Department of Commerce; 1983, An Assessment of U.S. Competitiveness in High Technology Industries, International Trade Administration. 